\title{
Primary hepatic angiosarcoma in a 64-year-old man: A case report
}

\author{
GEN CHEN ${ }^{*}$, JIANFENG LI* , RENHUA WAN, GUORONG WANG and JUN SHI
}

Department of General Surgery, The First Affiliated Hospital of Nanchang University, Nanchang, Jiangxi 330006, P.R. China

Received January 27, 2015; Accepted September 28, 2015

DOI: $10.3892 / 01.2016 .4277$

\begin{abstract}
Primary hepatic angiosarcoma (PHA) is rare, does not possess any characteristic tumor markers, is primarily observed in the elderly, and often presents with nonspecific symptoms, including discomfort or distension of the abdomen, weight loss and fatigue. Thus, PHA is difficult to diagnose, particularly if the patient presents no history of exposure to carcinogens, and its definitive diagnosis requires histological examination following surgery. Patients with PHA present poor long-term survival, and surgical resection of the tumor is currently the best treatment option for PHA. In the present report, the case of a 64-year-old man initially diagnosed with hydatid cyst, who was subsequently diagnosed with a giant PHA in the middle of the liver, is described. Further studies are required to investigate the diagnosis and treatment of PHA.
\end{abstract}

\section{Introduction}

Primary hepatic angiosarcoma (PHA) is a rare condition difficult to diagnose, particularly if the patient presents no history of exposure to carcinogens (1). PHA is primarily observed in the elderly, does not possess any specific tumor markers, and often presents with nonspecific symptoms, including discomfort or distension of the abdomen, weight loss and fatigue (2). To date, a limited number of cases of PHA have been reported in the English literature (3). In order to investigate the diagnosis and treatment options for PHA, the case of a 64-year-old man who was initially diagnosed with hydatid cyst and subsequently diagnosed with a giant PHA in the middle of the liver is described in the present study.

\section{Case report}

A 64-year-old man was admitted to the Department of General Surgery of The First Affiliated Hospital of Nanchang University (Nanchang, China) in May 2014 with a space-occupying lesion

Correspondence to: Professor Jun Shi, Department of General Surgery, The First Affiliated Hospital of Nanchang University, 17 Yong Wai Zheng Street, Nanchang, Jiangxi 330006, P.R. China E-mail: shijun6207@126.com

*Contributed equally

Key words: hepatic angiosarcoma, hepatosarcoma, surgery of the liver, as revealed by physical examination conducted $\sim 23$ days earlier. The patient had not experienced hepatalgia, nausea, vomiting, hematemesis or hematochezia since the onset of the lesions. The patient underwent an ultrasound (US; Philips iU22 Ultrasound System; Philips Healthcare, Andover, MA, USA) examination of the abdomen on May 10, 2014, which revealed the presence of a cystic mass in the liver. Thus, further examination was recommended, since the results of the US scan did not discard a possible hydatid cyst. In consequence, the patient underwent a full examination for parasites at the Jiangxi Provincial Institute of Parasitic Diseases (Nanchang, China) on May 16, 2014. The results of the analyses were negative for toxoplasma enzymes, schistosome immunity test, and cysticercosis and lung fluke enzyme-linked immunosorbent assay. Therefore, the patient was readmitted to The First Affiliated Hospital of Nanchang University for further treatment. On physical examination, the abdomen was soft, and tenderness was observed in the whole abdomen, but no rebound tenderness or palpable mass in the abdomen were detected. The patient did not present a history of use of intravenous drugs, tattoos, body piercing, excessive alcohol intake, obesity or previous work with toxic chemicals. In addition, the patient did not present a prior history of surgery, medical illnesses or known allergies, and was not receiving any medication. There was no significant family history of biliary or liver diseases.

Echocardiography demonstrated the left atrial to be enlarged $(41 \mathrm{~mm})$, and the ejection fraction of the ventriculus sinister to be $64 \%$. Abdominal magnetic resonance imaging (MRI; Magnetom ${ }^{\circledR}$ Trio Tim 3.0 MRI; Siemens AG, Munich, Germany) revealed a multiple cystic space-occupying lesion, measuring $\sim 10.3 \times 12.0 \mathrm{~cm}$, which originated from the middle of liver (Fig. 1A). On T2-weighted imaging (WI), the tumor was characterized by a medium-high intensity signal, which was clearly delineated from the surrounding liver tissue with internal septal structures separating the fluid-filled spaces (Fig. 1B), while on T1-WI, a low intensity signal was apparent within the cystic spaces (Fig. 1C).

The results of the laboratory tests were within the normal limits. The following blood tests conducted on the patient were all normal: Prothrombin time, $10.6 \mathrm{sec}$ (normal range, 9.8-12.1 sec); prothrombin time ratio, 0.91 (normal range, 0.85-1.15); international normalized ratio, 94\% (normal range, 70-130\%); activated partial thromboplastin time, $24.2 \mathrm{sec}$ (normal range, 22.7-31.8 sec); and thrombin time, $10.6 \mathrm{sec}$ (normal range, 14.0-21.0 sec). Urine tests revealed that $\mathrm{pH}$ and urine specific gravity were normal, 6.0 (normal range, 5.0-8.0) and 1.020 (normal range, 1.010-1.035), respectively. 
There was no protein, glucose, ketones, urobilinogen, bilirubin, leukocytes, occult blood or vitamin $\mathrm{C}$ in the urine. The patient was positive for hepatitis B surface antigen (HBsAg, $>250 \mathrm{IU} / \mathrm{ml}$; normal range, $0-0.050 \mathrm{IU} / \mathrm{ml}$ ), negative for hepatitis B surface antibody (HBsAb, $0.590 \mathrm{mIU} / \mathrm{ml}$; normal range, $0-10 \mathrm{mIU} / \mathrm{ml}$ ), negative for hepatitis $B$ envelope antigen (HBeAg, 0.074 PEI U/ml; normal range, 0-0.18 PEI U/ml), negative for hepatitis B envelope antibody $(0.020 \mathrm{~S} / \mathrm{CO}$; normal range, $>1 \mathrm{~S} / \mathrm{CO}$ ) and positive for hepatitis $\mathrm{B}$ core antibody (13.010 S/CO; normal range, $0-1 \mathrm{~S} / \mathrm{CO}$ ). In addition, the patient's leukocyte, erythrocyte and platelet levels were analyzed and were $6.26 \times 10^{9}$ cells $/ 1$ (normal range, 3.97-9.15 $\times 10^{9}$ cells $/ 1$ ), $3.42 \times 10^{12}$ cells $/ 1$ (normal range, $4.09-5.71 \times 10^{12}$ cells/l) and $98 \times 10^{9}$ cells/1 (normal range, $85-303 \times 10^{9}$ cells/1), respectively. The levels of serum carcinoembryonic antigen $(1.60 \mathrm{ng} / \mathrm{ml}$; normal range, $0-6.5 \mathrm{ng} / \mathrm{ml})$, carbohydrate antigen (CA)-199 (13.71 U/ml; normal range, 0-27.00 U/ml), CA-125 (10.35 U/ml; normal range, 0-35.00 U/ml) and $\alpha$-fetoprotein (AFP; $2.23 \mathrm{ng} / \mathrm{ml}$; normal range, $0-7.00 \mathrm{ng} / \mathrm{ml}$ ) were also normal. On the basis of these findings, the patient was diagnosed with liver lesions.

Next, the patient underwent an exploratory laparotomy with complex liver resection and cholecystectomy through a right back ' $\mathrm{L}$ ' incision. A large cyst-solitary mass located on the middle of the liver was detected, and subsequently excised. On macroscopic examination, the resected liver specimen was observed to contain a cystic-appearing mass measuring $10.5 \times 10.0 \times 4.0 \mathrm{~cm}$, and a solid-appearing mass measuring $8.0 \times 5.0 \times 3.5 \mathrm{~cm}$ (Fig. 2A). The thickness of the cyst wall was $0.2-0.5 \mathrm{~cm}$, and the gallbladder displayed chronic inflammation. Microscopically, the lumen was disordered and the vascular lumens anatomosed each other. Blood tumor-like vessels were present in certain areas, and lining cells of different sizes were observed, alongside abundant tumor cells that were located between the vessels. The cells contained abundant cytoplasm and oval or irregular nuclei. A number of tumor giant cells with unusual appearance and multiple nuclei were detected using hematoxylin and eosin (H\&E; Nanchang Rain Experiment Equipment Co., Ltd., Nanchang, China) staining (Fig. 2B and C; magnification, x200; Olympus BX41; Olympus Corporation, Tokyo, Japan). Immunohistochemistry demonstrated the tumor cells to be cytokeratin (mouse monoclonal; catalog no., MAB-0671), epithelial membrane antigen (mouse monoclonal; catalog no., Kit-0011), vimentin ${ }^{+}$ (Vim ${ }^{+}$; rabbit monoclonal; catalog no., RMA-0547), hepatocyte paraffin 1- (mouse monoclonal; catalog no., MAB-0249), glycine(rabbit polyclonal; catalog no., ab9442; Abcam, Cambridge, UK) smooth muscle actin ${ }^{+}\left(\mathrm{SMA}^{+}\right.$; mouse monoclonal; catalog no., Kit-0006) desmin- (mouse monoclonal; catalog no., Kit-0023), factor (F) VIII' (rabbit polyclonal; catalog no., RB-0070), actin ${ }^{+}$ (mouse monoclonal; catalog no., Kit-0032), cluster of differentiation (CD) $31^{+}$(mouse monoclonal; catalog no., MAB-0031), $\mathrm{CD}^{+} 4^{+}$(mouse monoclonal; catalog no., Kit-0004) in the vessels, and Ki-67 ( $60 \%$ locally; mouse monoclonal; catalog no., Kit-0005). All antibodies were purchased from Fuzhou Maixin Biotech., Co., Ltd. (Fuzhou, China) unless otherwise stated, and were ready to use. The dilution of Glycine (ab 9442) was 1:1,000. The surgical margins were negative, and a final diagnosis of PHA was established.

The second afternoon $\sim 50 \mathrm{~h}$ following surgery, the patient experienced abdominal distension and vomited yellow bile
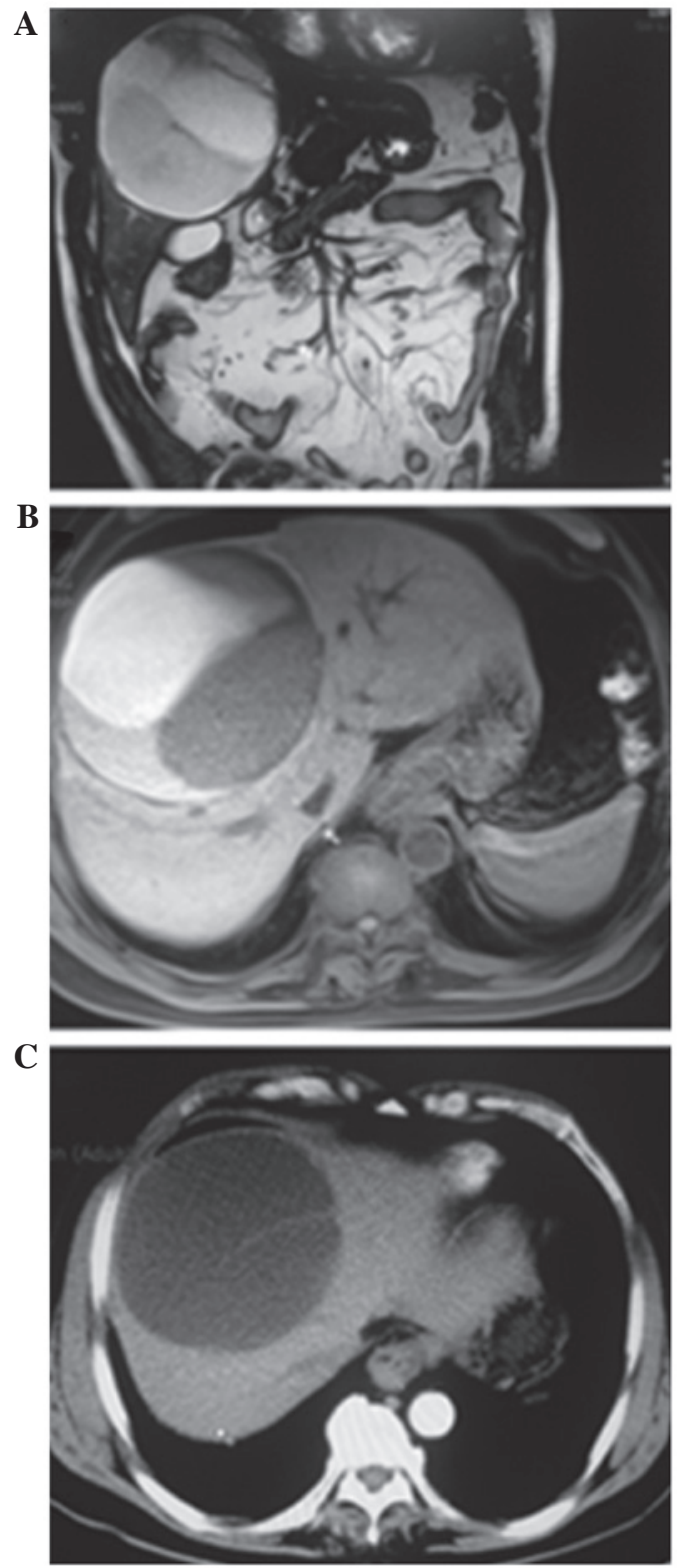

Figure 1. (A) Abdominal magnetic resonance imaging revealed multiple cystic lesions measuring $\sim 10.3 \times 12.0 \mathrm{~cm}$ originating from the center of liver. (B) T2-weighted imaging characterized the tumor with a medium-high intensity signal. (C) T1-weighted imaging demonstrated low signal intensity within the cystic spaces.

fluid-like liquid. In addition, the patient experienced dizziness when rising. The blood pressure was $126 / 83 \mathrm{mmHg}$, and the laboratory tests revealed levels of white blood cells $=14.4 \times 10^{9}$ cells $/ 1$, hemoglobin $=92 \mathrm{~g} / 1$, percentage of neutrophilic granulocyte $=88 \%$, alanine transaminase $=596 \mathrm{U} / 1$ and aspartate aminotransferase $=572 \mathrm{U} / \mathrm{l}$. Chest X-ray (Kodak Direct View DR5100; Kodak, Rochester, NY, USA) detected two patchy areas of high density in the lung field, suggestive of infection, in addition to elevation of the right diaphragm, presence of a liquid-gas shadow surrounding the liver and intestinal pneumatosis. In consequence, the patient received treatment to protect the liver, including administration of enema with glycerin and local application of mirabilite on the abdomen. That evening, at 11:30 p.m. (60 h following surgery), the patient was walking in the ward aided by other 
individuals when the patient suddenly lost consciousness. The vital signs of the patient were undetectable, and his pulmonary artery pulsatility and respiratory capacity disappeared. In consequence, cardiopulmonary resuscitation was immediately initiated, and the patient was administered Ringer's lactate solution and dicarbonate to expand the circulating blood volume and correct acidosis, dopamine to increase blood pressure, and adrenaline, lidocaine and atropine to promote cardiac resuscitation.

Following $80 \mathrm{~min}$, the patient did not exhibit noticeable cardiac rhythm or signs of breathing. Therefore, the patient was announced deceased.

\section{Discussion}

Angiosarcomas are malignant neoplasms of endothelial-type cells that line the walls of blood vessels, and account for $2-3 \%$ of all soft tissue sarcomas in adults. The primary sites for this type of tumor include skin, breast, soft tissues, bone and viscera (4). Among these, skin and breast are the most common sites for primary angiosarcomas (4). By contrast, PHAs are rare, and account for $<5 \%$ of all angiosarcomas $(4,5)$ and $1.8 \%$ of all hepatic malignancies (6). Accurate diagnosis of PHAs is difficult, since the majority of cases of PHA do not exhibit any obvious risk factors, and the common symptoms of PHA, including abdominal pain, fatigue and weight loss, are nonspecific (2). In the present study, the case of a 64-year-old man who presented a giant PHA located in the middle of the liver is reported.

The etiology of PHA remains unclear, although previous case-control studies have indicated that $\sim 1 / 3$ of all cases of PHA appear to be caused by exposure to environmental carcinogens, including thorium dioxide, arsenical insecticides or polyvinyl chloride. Exposure to these chemicals is rare, and the etiology of PHA remains unknown (7).

The diagnosis of PHA requires pathological analysis (8). Epithelioid hemangioendothelioma (EHE), a rare and usually low-grade malignant tumor, should be considered in the differential diagnosis of PHA (3). Compared with EHE, PHA displays limited stromal tissue and elongated or round tumoral endothelial cells with severe nuclear atypia and frequent mitoses, which grow along dilated sinusoids separated by surviving atrophic or hyperplastic hepatocytes $(9,10)$. Tumor markers such as CD31, CD34, podoplanin and FVIII-related antigen are often used in combination for the immunohistochemical diagnosis of angiosarcomas, since loss of expression of $\geq 1$ of these markers has been reported in $40 \%$ of tumors. In particular, CD31 and FVIII-related antigen has been suggested to be the most sensitive of the aforementioned combinations, since $90 \%$ of the cases of PHA previously studied were observed to express one of these two markers (11). In the present case report, immunohistochemical analysis demonstrated the tumor to be $\mathrm{CD} 31^{+}$, $\mathrm{FVIII}^{+}, \mathrm{CD} 34^{+}, \mathrm{Vim}^{+}$and $\mathrm{SMA}^{+}$.

The diagnosis of PHA is difficult, particularly if the patient does not present a history of exposure to carcinogens, since PHA is not characterized by any specific tumor marker (12). Morphologically, PHA may appear as multiple nodules, dominant masses, or a diffusely infiltrating lesion, and its appearance may vary slightly in computed tomography (CT) and MRI (13), being more complex if the patients are affected
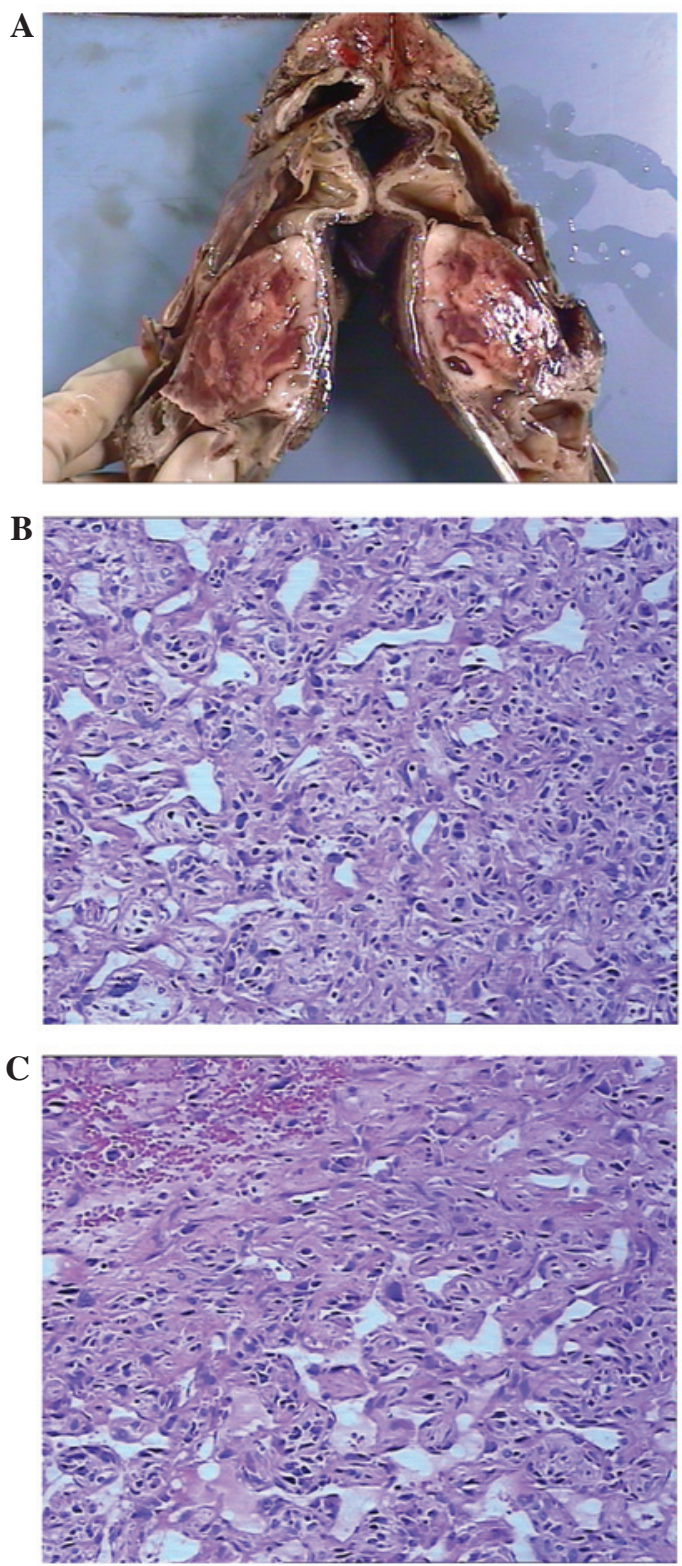

Figure 2. (A) Macroscopically, the resected liver specimen consisted of a cystic-appearing mass measuring $10.5 \times 10.0 \times 4.0 \mathrm{~cm}$ and a solid-appearing mass measuring $8.0 \times 5.0 \times 3.5 \mathrm{~cm}$. The cut surface was grey-white and red-gray. (B and C) Microscopic evaluation demonstrated that the vascular lumen was disordered and anastomosed each other. Part of the region had characteristics similar to hemangioma and the lining cells were various sizes. Tumor cells were observed between blood vessels. The cells had abundant cytoplasms with oval or irregular nuclei and there were multi-core giant tumor cells observed. Hematoxylin and eosin stain; magnification, x200.

by intratumoral hemorrhage, cirrhosis or exposure to toxic substances (3). Selective hepatic angiography in combination with dual-phase spiral CT may aid the diagnosis of PHA and facilitate the analysis of potential complications, since it may provide sufficient information about the main blood vessels in the liver and tumor $(14,15)$. In the present case, MRI revealed a multiple cystic space-occupying lesion, which measured $\sim 10.3 \times 12.0 \mathrm{~cm}$ and originated from the middle of the liver. On T1-WI, a low intensity signal was detected within the cystic spaces, while on T2-WI, the tumor exhibited a medium-high intensity signal, clearly delineated from the surrounding liver tissue. 
The long-term survival in patients with PHA is poor, due to the rapid progression of the disease, its high recurrence rate, and its resistance to traditional chemo- and radiotherapies (16-18). Currently, no formal guidelines exist for the treatment of PHA (19). PHA is a rare type of angiosarcoma, and is associated with poorer prognosis than other types of angiosarcomas. Patients with PHA do not exhibit any specific symptoms or signs, although spontaneous PHA with intraperitoneal hemorrhage is common, and may be fatal (3). Thus, the pathological diagnosis of PHA is essential. However, CT or US-guided fine-needle aspiration biopsy are dangerous and non-diagnostic procedures.

There are various treatment regimens for patients with PHA. However, due to the high recurrence rate and poor post-transplant survival rate of patients, liver transplantation is no longer provided (20). Kim et al (8) reported that a combination of chemotherapy resulted in an improved outcome for 2 out of 4 patients, suggesting the potential usefulness of palliative chemotherapy to improve the survival rate of patients. The initial first-line chemotherapy for 3 out of the 4 patients was 5-fluorouracil and carboplatin in combination with transhepatic artery infusion of doxorubicin. Following 2 cycles of treatment 2 patients succumbed to progressive disease (PD). The 3 rd patient received a second palliative ifosfamide/doxorubicin (IA) regimen chemotherapy composed of intravenous ifosfamide infusion every 3 weeks, accompanied by doxorubicin infusion via hepatic artery every 5 weeks for 5 cycles. Subsequently, the patient received 6 additional cycles of chemotherapy with ifosfamide and doxorubicin intravenously. The patient survived with stable disease for 16 months following diagnosis. The 4th patient was treated with IA regimen as the first-line chemotherapy and had stable disease for 4 months. Following 5 cycles, the patient developed PD and was administered with paclitaxel, which was replaced subsequent to the first cycle with bevacizumab. Finally, the patient succumbed to PD following the first cycle of bevacizumab, with an overall survival time of 9 months. Even though 2 patients succumbed $<3$ months following diagnosis, the remaining 2 patients received second or third lines of chemotherapy regimens and survived for 16 and 9 months, respectively (8).

Currently, the best treatment option for PHA is partial surgical resection of the liver to remove the tumor $(3,21)$. Zhou et al $(22)$ reported that out of 6 patients with PHA with solitary masses, 3 patients underwent right hepatectomy ( 2 patients survived for $>1$ year; 1 patient succumbed to disease perioperatively), 1 patient underwent extended right hepatectomy (survived for 6 months) and 2 patients underwent left hepatectomy (1 patient survived for 10 months; 1 patient was alive without recurrence 29 months later). In the present case report, the patient was initially diagnosed with hydatid cyst, and underwent an exploratory laparotomy with complex liver resection and cholecystectomy. Subsequently, the patient was diagnosed with a giant PHA in the middle of the liver, but suddenly passed away following surgery. Acute myocardial infarction was considered to be the direct cause of mortality, since the left atrial was enlarged and the blood pressure was high prior to the anesthesia. However, the exact cause of mortality remains unknown, since autopsy was refused by the patient's family.

In conclusion, in those cases of PHA where the liver tumors are supplied by multiple blood vessels, the possibility of antidiastole should be considered, particularly if the patient presents a long history of exposure to chemicals, no history of hepatitis or cirrhosis, and the immunohistochemistry results for tumor markers such as AFP are negative.

\section{Acknowledgements}

The authors would like to thank Mr. Hua Qiu (The First Affiliated Hospital of Nanchang University) for his important contribution to the manuscript.

\section{References}

1. Maluf D, Cotterell A, Clark B, et al Hepatic angiosarcoma and liver transplantation: Case report and literature review. Transplant Proc 37: 2195-2199, 2005.

2. Locker GY, Doroshow JH, Zwelling LA and Chabner BA: The clinical features of hepatic angiosarcoma: A report of four cases and a review of the English literature. Medicine (Baltimore) 58: 48-64, 1979.

3. Zheng YW, Zhang XW, Zhang JL, et al: Primary hepatic angiosarcoma and potential treatment options. J Gastroenterol Hepatol 29: 906-911, 2014.

4. Lahat G, Dhuka AR, Hallevi H, et al: Angiosarcoma: Clinical and molecular insights. Ann Surg 251: 1098-1106, 2010.

5. Fayette J, Martin E, Piperno-Neumann S, et al: Angiosarcomas, a heterogeneous group of sarcomas with specific behavior depending on primary site: A retrospective study of 161 cases. Ann Oncol 18: 2030-2036, 2007.

6. Alrenga DP: Primary angiosarcoma of the liver. Review article. Int Surg 60: 198-203, 1975.

7. Thomas LB and Popper H: Pathology of angiosarcoma of the liver among vinyl chloride-poly vinyl chloride workers. Ann N Y Acad Sci 246 (1 Toxicity of V): 268-277, 1975.

8. Kim HR, Rha SY, Cheon SH, et al: Clinical features and treatment outcomes of advanced stage primary hepatic angiosarcoma. Ann Oncol 20: 780-787, 2009.

9. Bioulac-Sage P, Laumonier H, Laurent C, Blanc JF and Balabaud C: Benign and malignant vascular tumors of the liver in adults. Semin Liver Dis 28: 302-314, 2008.

10. Cho NH, Lee KG and Jeong MG: Cytologic evaluation of primary malignant vascular tumors of the liver. One case each of angiosarcoma and epithelioid hemangioendothelioma. Acta Cytol 41: 1468-1476, 1997.

11. Rao P,Lahat G, Arnold C, et al: Angiosarcoma: A tissue microarray study with diagnostic implications. Am J Dermatopathol 35: 432-437, 2013.

12. Wang ZB, Yuan J, Chen W and Wei LX: Transcription factor ERG is a specific and sensitive diagnostic marker for hepatic angiosarcoma. World J Gastroenterol 20: 3672-3679, 2014.

13. Koyama T, Fletcher JG, Johnson CD, et al: Primary hepatic angiosarcoma: Findings at CT and MR imaging. Radiology 222: 667-673, 2002.

14. Rademaker J, Widjaja A and Galanski M: Hepatic hemangiosarcoma: Imaging findings and differential diagnosis. Eur Radiol 10: 129-133, 2000.

15. Park YS, Kim JH, Kim KW, et al: Primary hepatic angiosarcoma: Imaging findings and palliative treatment with transcatheter arterial chemoembolization or embolization. Clin Radiol 64: 779-785, 2009.

16. Maddox JC and Evans HL: Angiosarcoma of skin and soft tissue: A study of forty-four cases. Cancer 48: 1907-1921, 1981.

17. Almogy G, Lieberman S, Gips M, et al: Clinical outcomes of surgical resections for primary liver sarcoma in adults: Results from a single centre. Eur J Surg Oncol 30: 421-427, 2004.

18. Holden CA, Spittle MF and Jones EW: Angiosarcoma of the face and scalp, prognosis and treatment. Cancer 59: 1046-1057, 1987.

19. Chien CY, Hwang CC, Yeh CN, et al: Liver angiosarcoma, a rare liver malignancy, presented with intraabdominal bleeding due to rupture - a case report. World J Surg Oncol 10: 23, 2012.

20. Orlando G, Adam R, Mirza D, et al: Hepatic hemangiosarcoma: An absolute contraindication to liver transplantation - the European Liver Transplant Registry experience. Transplantation 95: 872-877, 2013.

21. Timaran $\mathrm{CH}$, Grandas $\mathrm{OH}$ and Bell JL: Hepatic angiosarcoma: Long-term survival after complete surgical removal. Am Surg 66: 1153-1157, 2000.

22. Zhou YM, Li B, Yin ZM, et al: Results of hepatic resection for primary hepatic angiosarcoma in adults. Med Sci Monit 16: CR61-CR66, 2010. 